\title{
Catégorisation agricole et transformations sociales
}

\section{Categorization in agriculture and social transformations}

Dans le cadre de l'Année internationale de l'agriculture familiale décidée par les Nations Unies en 2014, l'émergence de l'exploitation agricole familiale comme catégorie sociopolitique a mobilisé 1'attention (Sourisseau, 2014 ; Cochet, 2015). Au-delà la posture pragmatique d'action publique et de recherche visant à identifier et à accompagner spécifiquement une forme de production, se posent de nombreuses questions sur les fondements de la démarche de catégorisation, sur son efficacité, mais aussi, plus largement, sur les effets divers du ciblage de systèmes, de populations, d'instruments et de territoires par les politiques agricoles et rurales: effets territoriaux (aménagement, environnement), socio-économiques (inclusion/exclusion, construction) et politiques (redéfinition du champ de l'action publique).

À travers la présentation d'études de cas couvrant des terrains, des processus et des effets variés, le numéro thématique des Cahiers Agricultures «Catégorisations politiques et transformations sociales en agriculture» (Ancey et al., 2017) traite des questions du ciblage et de la catégorisation en agriculture, non seulement au travers des opérations techniques et des politiques de sélection, d'exclusion ou de mise en évidence de sous-groupes donnés au sein d'une population, mais également au travers des processus de transformation sociale. Plus largement, il interroge l'évolution des normes et des catégories en questionnant, entre autres, la montée en puissance des catégories territoriales, le ciblage des populations et des zones, et la légitimation des instruments privés de l'action publique.

\section{Deux principes de catégorisation}

Depuis la publication du rapport de la Banque mondiale (2008) sur le développement dans le monde, la question de l'agriculture et, plus largement, des territoires ruraux, a acquis une visibilité nouvelle dans le champ de l'action publique. Les politiques agricoles, les politiques de développement rural, la multiplication des actions et des discours politiques, comme l'implication de la société civile et des organisations internationales, notamment en Afrique via l'aide au développement, concourent à renforcer l'idée qu'il existerait une catégorie socio-politique ayant des problèmes spécifiques, auxquels il s'agirait de répondre par des mesures adéquates. Mais qu'est-ce que la catégorisation en agriculture? Quels en sont les effets géographiques et sociaux?

Dans l'ensemble des textes de ce numéro thématique, le ciblage n'est pas traité comme une donnée, c'est-à-dire une catégorie indépendamment de ceux qui la formulent, mais comme un enjeu de réflexion pour l'action publique. Il s'agit en particulier d'identifier les processus de catégorisation à l'œuvre dans la construction de la catégorie «agricole» dans différents contextes et les conditions de leur mise en œuvre, et de s'interroger sur les effets du ciblage. Quelle qu'en soit la forme (ciblage social/ciblage territorial), la catégorisation peut être assimilée à un acte performatif, en ce sens qu'elle peut influencer le cours de l'action publique, légitimer son champ et ses limites, influencer la conduite des acteurs cibles (les agriculteurs) et les dynamiques des territoires.

C'est autour de deux principes que les travaux proposés dans ce dossier invitent à mettre en perspective la question de la catégorisation, de sa construction et de ses effets.

Tout d'abord, la création politique de catégories peut procéder d'un "simple décalque» d'une catégorisation préexistante formalisée par l'action publique dans un contexte particulier et transférée, voire "plaquée», dans un autre contexte géographique. C'est notamment le cas en Europe centrale, où la Politique agricole commune a imposé aux pays qui ont rejoint l'Union européenne (UE) en 2004 et 2007, une catégorie particulière de «l'exploitation familiale», élaborée sur le modèle des structures agricoles occidentales. Le ciblage des aides découlant de cette catégorie a alors exclu une part très importante des familles paysannes. Ainsi, dans sa propre volonté d'uniformisation sociale de l'agriculture, qui considère implicitement que les plus petites exploitations sont vouées à une disparition prochaine, l'UE a favorisé des effets d'exclusion, marqués, entre autres, par un repli des populations rurales vers un modèle agricole vivrier (Maurel et al., 2003).

La catégorisation peut également résulter de l'émergence de nouveaux référentiels d'action publique, avec l'intégration de financements privés et de nouveaux acteurs (ONG, entreprises, bailleurs de fonds, etc.) et, plus largement, une perte de repères de la politique publique. À noter que d'autres formes émergentes d'auto-catégorisation, liées à des revendications politiques endogènes, par exemple, communautaires en Amérique Latine, ne sont pas abordées dans ce numéro.

\section{Action publique: changement et multiplication des référentiels}

Les textes présentés dans ce numéro thématique insistent sur les changements et la multiplication des référentiels. Trois principaux enseignements peuvent en être tirés : 
- tout d'abord, la catégorisation peut produire de l'exclusion et donc de l'invisibilité. Le ciblage de certaines formes et structures agricoles a pu écarter une part importante des populations paysannes des aides financières directes et des programmes d'aide à la modernisation et, paradoxalement, des programmes destinés aux zones défavorisées. Dans la marge ainsi produite (sociale, territoriale, etc.) se produisent néanmoins des transformations sous l'effet d'autres interventions, privées, ou de dynamiques locales ; - avec le changement d'échelle de l'action publique et des enjeux autour des questions de développement, les politiques agricoles, généralement nationales, ont pu s'associer à des politiques locales légitimées par des procédures de décentralisation. Des lors, des acteurs rendus invisibles dans les politiques nationales ont pu devenir des cibles explicites des enjeux territoriaux;

- enfin, le ciblage des populations est devenu une procédure omniprésente des politiques de lutte contre la pauvreté. Il tient d'ailleurs souvent lieu de programme de développement depuis le début des années 1980. Le ciblage des groupes - à risques, vulnérables, résilients, etc-comme tactique de gouvernement, relevant du champ politique autant que scientifique, a été étudié dans son principe (Castel, 1981 ; Foucault, 1994), dans ses méthodes et dans ses effets (Banque mondiale, 1998 ; Desrosières, 2010).

C'est à travers quatre études de cas en Europe centrale, Afrique et Inde que cette question de la catégorisation est analysée ici.

Pour Marie-Claude Maurel (2016), l'adhésion à l'UE des pays d'Europe centrale s'est faite au prix de l'exclusion explicite de catégories d'agriculteurs. À la suite du processus de privatisation, la plupart des formes sociales agricoles de ces pays, telles les petites exploitations de semi-subsistance ou les grandes exploitations sociétaires, se sont confrontées aux formes existantes dans les anciens États membres de l'Union européenne. Fondé sur une catégorie normative de l'exploitation familiale, le «modèle agricole européen»s'est alors révélé inapproprié dans des pays où les exploitations familiales moyennes et grandes n'existaient presque pas. L'article examine les effets distributifs pervers de l'application d'une politique de soutien (le régime simplifié de paiements directs) à des structures d'exploitation pour lesquelles elle n'a pas été conçue.

Pour Christine Lutringer (2017), le système d'approvisionnement public et de prix minimum de soutien, mis en place dans les années 1960 dans le secteur agricole de l'Inde comme volet stratégique de l'intervention publique, joue toujours un rôle pivot dans la politique de sécurité alimentaire. Les prix de soutien influencent les décisions des paysans sur le choix des différentes variétés et types de production. Concrètement, cette politique a conduit à un ciblage sur le blé et le riz, au détriment d'autres cultures comme les légumineuses et les oléagineux. Et les débats sur les réformes en cours, portant sur un ciblage plus explicite des catégories de paysans et des régions, privilégient l'argument central de l'efficacité économique du système productif agricole plutôt que celui de la justice sociale.

Vincent Ribier et Jean-Jacques Gabas (2016) montrent un paysage institutionnel du financement de l'agriculture en Afrique de l'Ouest en cours de recomposition, avec une multiplication et une diversification des acteurs ainsi qu'une imbrication croissante des financements publics et privés, où les fonds publics sont censés jouer un effet de levier. Cette évolution interroge la capacité des États à cibler les financements pour répondre aux grands enjeux de développement tels que la sécurité alimentaire, la réduction de la pauvreté et la modernisation de l'agriculture. Une étude comparative dans trois pays d'Afrique de l'Ouest (Sénégal, Côte d'Ivoire, Ghana) permet de montrer que les institutions nationales sont généralement trop faibles pour canaliser les nouveaux flux de financement vers les objectifs affichés de la politique agricole. Les disparités de financement du secteur agricole, déjà marquées par le passé, s'en trouvent exacerbées et l'accroissement global du montant de ces financements s'accompagne de la concentration de ceux-ci au profit de l'agriculture entrepreneuriale, au détriment de l'agriculture familiale, des biens publics et de certaines régions enclavées.

Enfin, en Afrique saharienne, Véronique Ancey (2016) montre que l'inadéquation des politiques nationales et des actions d'urgence internationales aux spécificités des populations pastorales renvoie aux difficultés historiques des administrations à prendre en compte la mobilité des troupeaux et la gestion commune des ressources naturelles. «L'invisibilité » des pasteurs s'est construite à travers des schémas de pensée sous-jacents aux systèmes d'information conçus pour des productions et des régions agricoles. D'un autre côté, la stratégie d'évitement des populations pastorales, qui leur a historiquement permis d'échapper aux contraintes exercées par l'administration, les dessert aujourd'hui en compromettant leur capacité à peser sur des débats fondamentaux au sein de leurs pays.

\section{Conclusion}

La catégorisation constitue un enjeu de pouvoir stratégique dans le développement des sociétés rurales. Les groupes minorisés sont confrontés à un dilemme que reflètent bien les débats dans les sciences sociales: adopter les identités collectives assignées (par exemple, ethnique au Sud, socioprofessionnelle au Nord), la terminologie et éventuellement le traitement correspondant, ne serait-ce que pour tenter de les requalifier, au risque de se trouver dans une impasse à cause d'une catégorie réifiante ou d'une politique versatile; ou bien s'en détacher pour produire d'autres identifications, par une codification constamment renouvelée des différences (Barth, 1969). Cette question de stratégie politique posée à certaines catégories sociales (notamment les agriculteurs) s'impose également aux sciences sociales avec d'autres enjeux: la responsabilité des chercheurs face à la politique des identités et la construction de leurs propres objets de recherche face aux catégorisations.

\section{Références}

Ancey V. 2016. Comment se construit l'invisibilité des populations pastorales au Sahel : l'expérience de la crise alimentaire de 2005 au Niger. Cahiers Agricultures 25(5): 55003. DOI: 10.1051/cagri/ 2016041.

Ancey V, Chevalier P, Sourisseau JM. 2017. Catégorisations politiques et transformations sociales en agriculture. Cahiers 
Agricultures, numéro thématique. Disponible sur http://www. cahiersagricultures.fr/fr/component/toc/?task=topic\&id=672.

Banque mondiale. 1998. Assessing Aid. What works, what does not, and why. Washington: Banque mondiale.

Banque Mondiale. 2008. L'agriculture au service du développement. Rapport sur le développement dans le monde. Washington: Banque mondiale, $36 \mathrm{p}$.

Barth F. 1969. Introduction. Ethnic groups and boundaries. The social organization of culture difference. Illinois: Waveland Press.

Castel R. 1981. La gestion des risques : de l'anti-psychiatrie à l'aprèspsychanalyse. Paris: Éditions de Minuit.

Cochet H. 2015. Controverses sur l'efficacité économique des agricultures familiales: indicateurs pour une comparaison rigoureuse avec d'autres agricultures. Revue Tiers Monde 1: 9-25.

Desrosières A. 2010. La politique des grands nombres : histoire de la raison statistique. Paris: La Découverte, $462 \mathrm{p}$.

Foucault M. 1994. Dits et écrits, tome 3: 1976-1979. Paris: Gallimard.

Lutringer C. 2017. Le ciblage des politiques agricoles en Inde: les implications du système d'approvisionnement public. Cahiers Agricultures 26(4). DOI: 10.1051/cagri/2017027.
Maurel MC. 2016. Penser la catégorisation des formes sociales de production agricole en Europe centrale. Cahiers Agricultures 25(4): 45002. DOI: $10.1051 /$ cagri/2016028.

Maurel MC, Halamska M, Lamarche H. 2003. Le Repli paysan. Trajectoires de l'après communisme en Pologne. Paris: L'Harmattan, $252 \mathrm{p}$.

Ribier V, Gabas J-J. 2016. Vers une accentuation des disparités dans le financement de l'agriculture en Afrique de l'Ouest? Cahiers Agricultures 25(6): 65007. DOI: 10.1051/cagri/2016045.

Sourisseau J-M. 2014. Agricultures familiales et mondes à venir. Versailles: Éditions Quae.

\section{Véronique Ancey ${ }^{1, *}$, Pascal Chevalier ${ }^{2}$, Jean-Michel Sourisseau}

${ }^{1}$ CIRAD, UMR Art-Dev, TA C-113/15, 73 rue Jean-François Breton, 34398 Montpellier Cedex 5, France

${ }^{2}$ Université Paul Valéry Montpellier 3, UMR Art-Dev, Route de Mende, 34199 Montpellier Cedex 5, France

* veronique. ancey@cirad.fr 